\title{
An Easy Approach to Increase the Precision of EBSD Analysis - Examples from a Sea Urchin Calcite Study
}

\author{
A. Goetz ${ }^{1, a}$, E. Griesshaber ${ }^{1, b}$ and W. W. Schmahl $1^{1, c}$ \\ ${ }^{1}$ Department für Geo- und Umweltwissenschaften, Ludwig-Maximilians-Universität München, \\ München, Germany \\ aandreas.goetz@Irz.uni-muenchen.de, ${ }^{\mathrm{b}}$ e.griesshaber@Irz.uni-muenchen.de, \\ cwolfgang.schmahl@Irz.uni-muenchen.de
}

Keywords: EBSD, high angular resolution, calcite, biomineralization, sea urchin spine

\begin{abstract}
Sea urchins mineralize Mg-calcite skeletons, both, within their exoskeletons as well as in their spines. In this study we have investigated sea urchin spines of the species Amblypneustes pachistus. The spines are round and consist of several wedges that extend from the base to the tip of the spine. The wedges are connected to each other by porous calcite. Rocking curves of the spines show a distribution of $0.5^{\circ}$ of the 110 reflection, with the domains being misoriented by $0.1^{\circ}$ to each other. In our EBSD system the average mean angular (MAD) deviation is $0.3^{\circ}$. This is higher than the signal that is needed for the detection of small misorientations of domains within a sea urcin spine.

In order to increase the precision (not the accuracy) of the EBSD measurements several factors, such as geometric artifacts and charging of calcite were minimized. Handling of these factors and utilizing the subsequently described statistical approach allowed for the identification of different domains within the spines. It further allowed calculating the degree of misorientation between these domains. Our EBSD analyses and the subsequent evaluation of the data show that the wedges forming the spines of Amblypneustes pachistus are mosaic crystals. The misorientation of the wedges to each other increases from the base of the spine towards its tip.

The here proposed method for increasing the precision of the angular resolution showed reproducibility on silicon of $0.05^{\circ}$.
\end{abstract}

\section{Introduction}

Sea urchins are marine animals that predominantly live on the sea floor. Their size ranges from several centimeters to a few tenths of centimeters. A sea urchin consists of a round, nearly spherically shaped, calcitc exoskeleton (consisting of ten plates) onto that several concentrically arranged spines are attached. These spines have a wide range of sizes, shapes and architectures. They all have in common that they appear to be single crystals not only in polarization microscopical studies, but also under all kinds of diffraction methods [e.g. 1, 2, 3, 4, 5, 6, 7] - a highly surprising fact. Sea urchin spine microstructure is highly porous and is called stereom (Fig. 1). In live animals it is filled with liquids. The single crystalline, highly porous stereom is often surrounded by a polycrystalline calcitic cortex [8].

The spines investigated in this study came from the sea urchin species Amblypneustes pachistus. These animals mainly feed on algae, seaweed and live close to the coastal shore. The analyzed individuum was found in a depth of $2 \mathrm{~m}$ close to Port MacDonnel, Southern Australia [9].

The highly porous exoskeleton and the spines of sea urchins are known to be low-Mg calcite single crystals. The investigated species builds spines that consist of massive wedges connected to each other by porous calcite (Fig. 1). The entire spine behaves as a single crystal, with its c-axis being parallel to its longitudinal axis. 

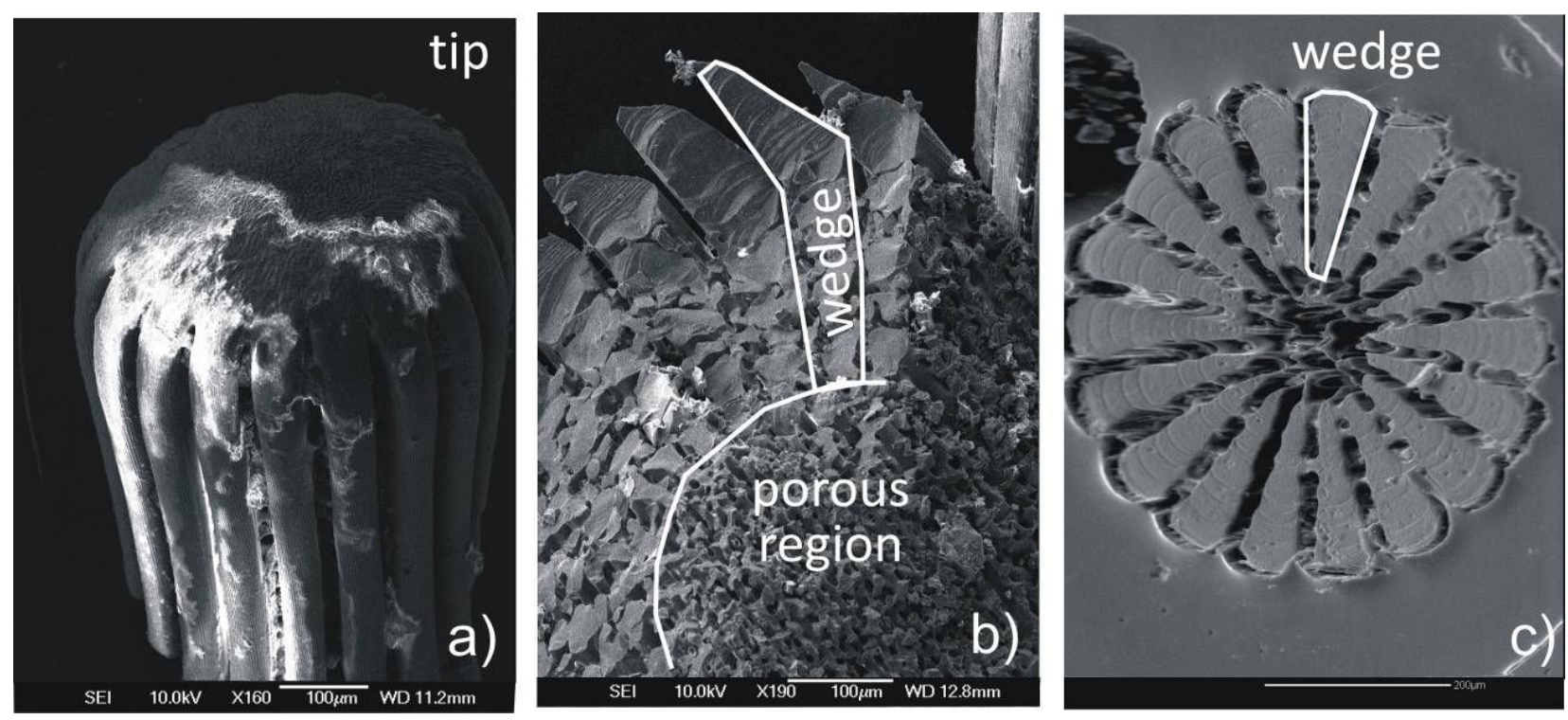

Figure 1: SEM images of the sea urchin spines. Figure 1a shows the unbroken surface and the tip of the spine. Figure $1 \mathrm{~b}$ highlights the fracture surface and the interior of a spine. One can distinguish the wedges at the outer portion and the porous region within the core of the spine. Figure 1c depicts a polished transverse section of the spine, similar to the section used for EBSD analysis.

This study gives a means to increase the precision of EBSD data sets. So far there are only two other suggestions in literature, that follow similar targets. One is known as the "pattern shift method" (e.g. [10],[11],[12]) and is based on the comparison of two Kikuchi patterns and the calculation of the amount of the shift of these two patterns relative to each other. The other method is based on indexing of the patterns in a three-dimensional Hough space [13]. This method has so far been applied to calculated texture data sets.

\section{Samples}

We analysed spines of Amblypneustes pachistus, obtained from the Zoologische Staatssammlung München, Germany (Reg. Nr. 20060657). For XRD analysis the spine was mounted with its longitudinal axis (crystallographic c-axis) perpendicular to the X-ray beam.

For EBSD analysis sample surfaces were highly polished. The final polish was performed with a 0.25 micron diamond polish. Subsequently, the samples were etched with a suspension of aluminium nanoparticles for at least 45 seconds and coated with 5-10 nm carbon. The cuts through the spines were done parallel and perpendicular to the longitudinal axis of the spines.

The measurements on silicon were performed on a single crystalline Si-wafer, the EBSD standard of HKL Technologies.

\section{Methods}

Instrumentation. Rocking curves were obtained on a Stoe powder diffractometer using Mo-K $\alpha_{1}$ radiation and performing omega scans of the 110 - reflection of the sea urchin spines. The step size was $0.01^{\circ}$, the detector was protected from the strong reflection by several layers of aluminium foil.

The EBSD patterns were recorded in a field emission gun SEM (JEOL JSM $6500 \mathrm{~F}$ ) with an accelerating voltage of $20 \mathrm{kV}$ under a tilt angle of $70^{\circ}$. The Kikuchi patterns were recorded with a NORDLYS detector and were indexed with the CHANNEL 5 software package, Hough space resolution was 95. The average MAD (mean angular deviation) during the measurement was $0.3^{\circ}$. Any measurements with MAD above $0.5^{\circ}$ were not used.

SEM images were obtained in the same microscope with an accelerating voltage of $10 \mathrm{kV}$. 
High precision EBSD measurements. The attempt to use a statistical approach is promising as there is a statistical distribution within the multitude of indexed Kikuchi patterns (Fig. 2c). However, in order to achieve even higher precision regarding the angular resolution of the EBSD data several errors during data collection had to be eliminated:

1. Geometrical misfits between beam, sample and detector.

2. Surface charging of the non-conductive calcite.

3. The aspect ratio of the detector should not change during a measurement cycle.

To eliminate the influence of the above stated factors, we analysed several small maps $\left(10^{*} 10\right.$ pixels, 1.5 micrometer step size). This step size turned out to be a good compromise between charging of calcite and a good spatial resolution. After every map, the stage was driven parallel to the tilt axis by 15 micrometers and a new map was analysed. Movements perpendicular to the tilt axis were performed with a constantly kept focus.

The obtained data sets were evaluated with the algebra program MATHEMATICA. As a first step the average orientation of the complete data set was calculated, using all indexed points with a MAD lower than $0.5^{\circ}$. To avoid the mathematically non-trivial normalization of the orientation matrix, the average orientation of the a- and c-axes was calculated independently from each other. The analysis point that is closest to the average orientation of both axes, was then defined as the average orientation. The error induced is usually two orders of magnitudes lower than the resulting angular resolution. After the first definition of the average orientation, "wild spikes" were removed by discarding all points that were not within three times the standard deviation of the average orientation. The rest of the dataset (usually more than $95 \%$ of the entire data set) was then used to determine again the average orientation of the entire data set.

The average orientation of the data sets was compared and small orientational differences in the range around $0.1^{\circ}$ could be observed. In order to obtain this it is necessary that the analyses have a stochastic distributional pattern of the orientation. That this is the case is demonstrated in Fig. 2.
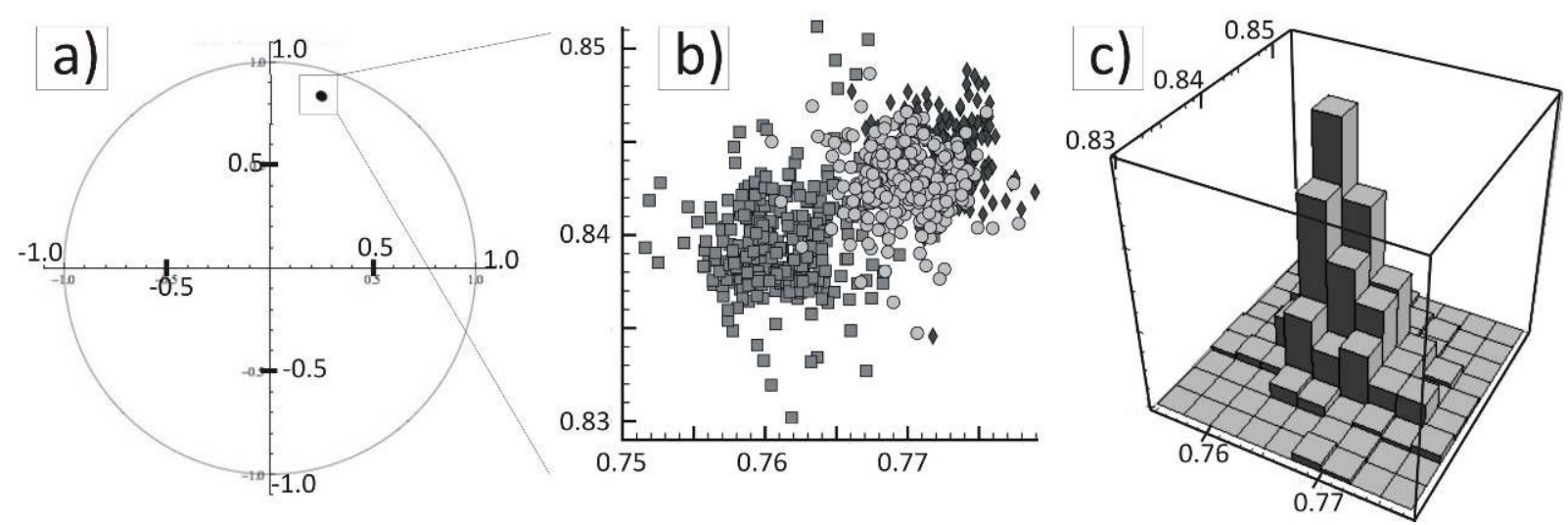

Figure 2. In Figure $2 \mathrm{a}$ the $<001>$ pole figure of the complete dataset of line 1 of Figure 3 is shown. The scale of the pole figure is the distance from the center on a unit circle. Figure $2 b$ gives an enlarged portion of Figure 2a. The squares correspond to wedge 1, the diamonds to wedge 2 and the circles to wedge 3 . Figure $2 \mathrm{c}$ shows a $3 \mathrm{D}$ histogram of the squares (line 1) and highlights well the statistical distribution pattern of the data points. 


\section{Results and Discussion}

XRD. Rocking curves (= omega scans) of the [110]-reflection were measured on different parts of the sea urchin spines. They showed that the single crystal spine consists of different mosaic domains that have a maximum misorientation of $0.5^{\circ}$. The domains are misoriented by about $0.1^{\circ}$ towards each other (Fig. 3). The mosaicity is getting more pronounced towards the tip of the spine.
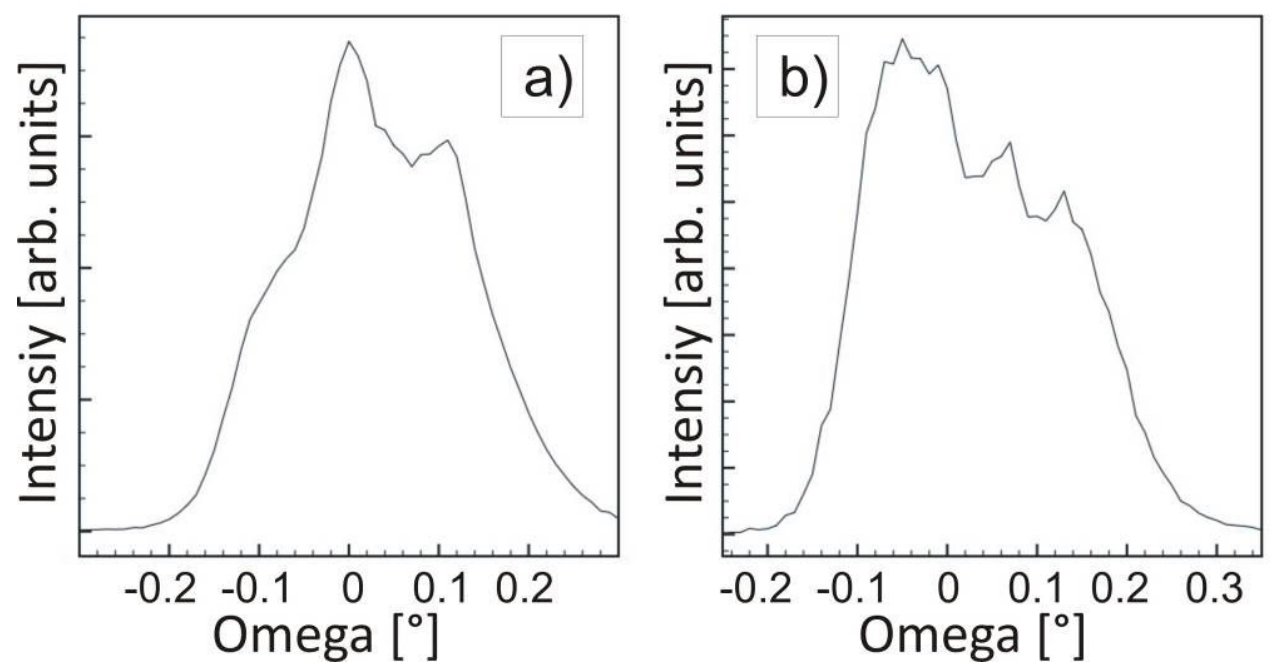

Figure 3. 110 rocking curves (omega scans) of a sea urchin spine is shown in Figures $3 \mathrm{a}$ and $3 \mathrm{~b}$. The range of misorientation of the domains in a spine is around 0.5 degrees. Domains that are tilted by $0.1^{\circ}$ can be easily distinguished from each other. Figure 3 a shows the rocking curve that has been measured close to the base of the spine, while Figure $3 \mathrm{~b}$ gives an example of a rocking curve that has been analysed close to the tip of the spine. The mosaicity of the material is getting more pronounced at the tip of the spine.

EBSD on silicon. For evaluating the limitations of the described method, we also have measured eight EBSD data sets under the above described conditions on the silicon reference of HKL. These maps never showed misorientations higher than $0.05^{\circ}$ from each other. On average the misorientation of the EBSD maps between each other was $0.03^{\circ}$.

EBSD on sea urchin spines. For this study, sea urchin spines were analyzed in transverse and in longitudinal sections. Figure 4 shows the results of one line on a transversal section through the spine. It is clearly visible that single wedges have similar general orientations, but are slightly tilted relative to each other by 0.2 to $0.6^{\circ}$. The misorientation angles between all the maps are shown in detail in Table 1. The corresponding pole figure of all measured data points is shown in Figure 2.

The dataset on the transversal section through the spine gives orientational information from three wedges (Figure 4). The average misorientation between the wedges is $0.35^{\circ}, 0.5^{\circ}$ and $0.55^{\circ}$. 

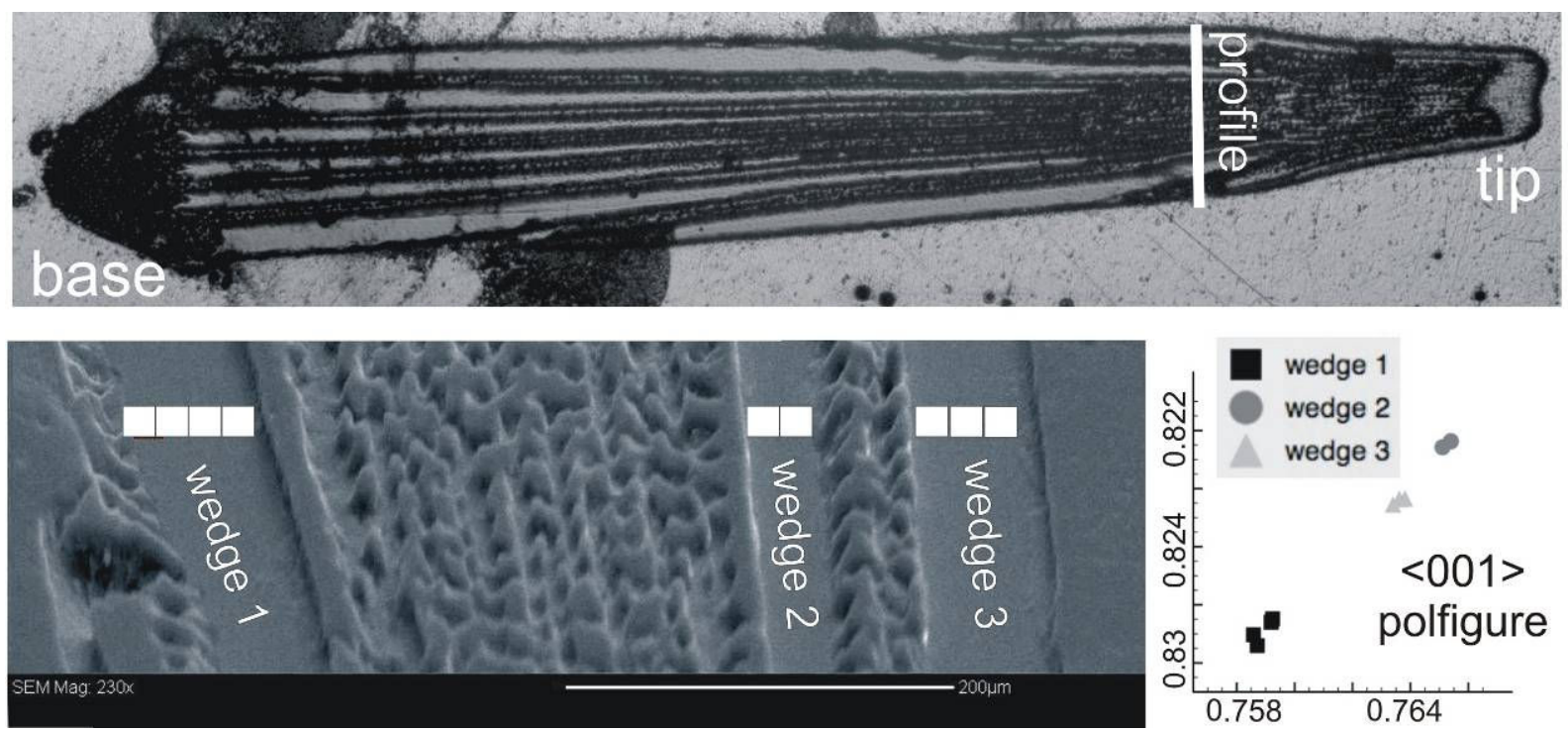

Figure 4. The upper part of Figure 4 shows a longitudinal cut through the spine. The exact location of the conducted EBSD is shown in detail in the lower image. The $<001>$ pole figure (scale see Figure 3) is shown on the right. It is obvious that each wedge has a distinct orientation. The misorientations of the maps relative to each other are given in Table 1.

Table 1. Misorientation between the EBSD maps measured along a profile on the longitudinal cut (Figure 4) of a sea urchin spine. Maps 1 to 4, then maps 5 and 6 and maps 7 to 9 are located on the same wedge (wegde1, wedge2, wedge3), respectively.

\begin{tabular}{|c|cccc|cc|ccc|}
\cline { 2 - 9 } \multicolumn{1}{c|}{} & Map 1 & Map 2 & Map 3 & Map 4 & Map 5 & Map 6 & Map 7 & Map 8 & Map 9 \\
\hline Map 1 & $0^{\circ}$ & $0.05^{\circ}$ & $0.03^{\circ}$ & $0.06^{\circ}$ & $0.57^{\circ}$ & $0.6^{\circ}$ & $0.43^{\circ}$ & $0.4^{\circ}$ & $0.4^{\circ}$ \\
Map 2 & $0.05^{\circ}$ & $0^{\circ}$ & $0.08^{\circ}$ & $0.08^{\circ}$ & $0.62^{\circ}$ & $0.64^{\circ}$ & $0.47^{\circ}$ & $0.44^{\circ}$ & $0.44^{\circ}$ \\
Map 3 & $0.03^{\circ}$ & $0.08^{\circ}$ & $0^{\circ}$ & $0.06^{\circ}$ & $0.54^{\circ}$ & $0.57^{\circ}$ & $0.41^{\circ}$ & $0.37^{\circ}$ & $0.37^{\circ}$ \\
Map 4 & $0.06^{\circ}$ & $0.08^{\circ}$ & $0.06^{\circ}$ & $0^{\circ}$ & $0.58^{\circ}$ & $0.6^{\circ}$ & $0.46^{\circ}$ & $0.42^{\circ}$ & $0.42^{\circ}$ \\
\hline Map 5 & $0.57^{\circ}$ & $0.62^{\circ}$ & $0.54^{\circ}$ & $0.58^{\circ}$ & $0^{\circ}$ & $0.03^{\circ}$ & $0.24^{\circ}$ & $0.23^{\circ}$ & $0.23^{\circ}$ \\
Map 6 & $0.6^{\circ}$ & $0.64^{\circ}$ & $0.57^{\circ}$ & $0.6^{\circ}$ & $0.03^{\circ}$ & $0^{\circ}$ & $0.27^{\circ}$ & $0.26^{\circ}$ & $0.26^{\circ}$ \\
\hline Map 7 & $0.43^{\circ}$ & $0.47^{\circ}$ & $0.41^{\circ}$ & $0.46^{\circ}$ & $0.24^{\circ}$ & $0.27^{\circ}$ & $0^{\circ}$ & $0.06^{\circ}$ & $0.06^{\circ}$ \\
Map 8 & $0.4^{\circ}$ & $0.44^{\circ}$ & $0.37^{\circ}$ & $0.42^{\circ}$ & $0.23^{\circ}$ & $0.26^{\circ}$ & $0.06^{\circ}$ & $0^{\circ}$ & $0^{\circ}$ \\
Map 9 & $0.4^{\circ}$ & $0.44^{\circ}$ & $0.37^{\circ}$ & $0.42^{\circ}$ & $0.23^{\circ}$ & $0.26^{\circ}$ & $0.06^{\circ}$ & $0^{\circ}$ & $0^{\circ}$ \\
\hline
\end{tabular}




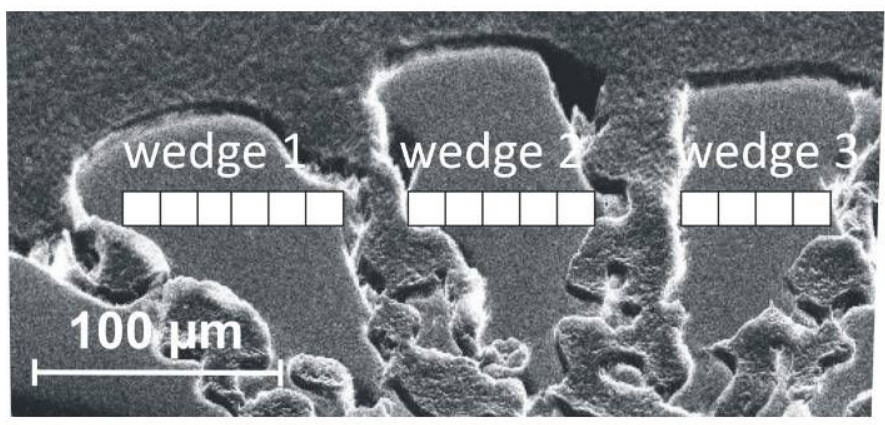

\begin{tabular}{|c|ccc|}
\cline { 2 - 4 } \multicolumn{1}{c|}{} & wedge & wedge & wedge \\
\hline wedge 1 & $0.1^{\circ}$ & $0.35^{\circ}$ & $0.55^{\circ}$ \\
wedge 2 & $0.35^{\circ}$ & $0.05^{\circ}$ & $0.3^{\circ}$ \\
wedge 3 & $0.55^{\circ}$ & $0.3^{\circ}$ & $0^{\circ}$ \\
\hline
\end{tabular}

Figure 4. EBSD data sets (maps) measured on three wedges of a sea urchin spine. The average misorientation between the wedges is shown in the table on the right hand side.

\section{Conclusions}

We can improve the reproducibility and thus the precision of the EBSD data 10 to 20 times in comparison to the MAD (mean angular deviation) of the silicon standard. It is important to note that this is only an increase in precision and not in accuracy.

The datasets on the sea urchin spines show that there is a misorientation of the wedges towards each other. The absolute value of this misorientation is between 0.3 and $0.6^{\circ}$ and fits well to the observed mosaicity observed in XRD rocking curves.

\section{References}

[1] W.J. Schmidt: Zool. Jb. Vol. 47 (1930), p. 357-510.

[2] D.M. Raup, : The endoskeleton. In Physiology of Echinodermata, edited by R.A. Boolootian, Wiley Interscience Publishers, New York (1966), p. 379-395.

[3] G. Donnay and D.L. Pawson: Science Vol. 166 (1969), p. 1147-1150.

[4] J.D. Currey and D. Nichols: Nature Vol. 214 (1967), p. 81-83.

[5] J. Paquette and R.J. Reeder: Am. Min. Vol. 75 (1990), p. 1151-1158.

[6] X. Su S. Kamat and A.H. Heuer: Jour. of Mat. Science Vol. 35 (2000), p. 5545-5551.

[7] U. Magdans and H. Gies: Eur. J. of. Min. Vol. 16 (2004), p. 261-268.

[8] K. Märkel and U. Röser: Zoomorphology Vol. 103 (1983), p. 25-41.

[9] Zoologische Staatssammlung München, Reg. Nr. 20060657.

[10] A.J. Wilkinson, G. Meaden and D.J. Dingley: Mat. Science and Technology Vol. 22 (2006), p. 1271-1278.

[11] A.J. Wilkinson, G. Meaden and D.J. Dingley: Ultramicroscopy Vol. 106 (2006), p. 307-313.

[12] X.D. Tao and A. Eades: Microscopy and Microanalysis Vol. 11 (2005), p. 341-353.

[13] C. Maurice and R. Fortunier: Journal of Microscopy (Oxford) Vol. 230 (2008), p. 509-520. 